\title{
Brown's Theorem and its Application for Enumeration of Dissections and Planar Trees
}

\author{
Evgeniy Krasko Alexander Omelchenko \\ Saint Petersburg Academic University \\ Saint Petersburg, Russia \\ $\{$ krasko.evgeniy, avo.travel\}@gmail.com
}

Submitted: Feb 20, 2014; Accepted: Jan 12, 2015; Published: Jan 27, 2015

Mathematics Subject Classifications: 05C30, 05A15

\begin{abstract}
We present new functional equations connecting the counting series of plane and planar (in the sense of Harary and Palmer) dissections. Simple rigorous expressions for counting symmetric $r$-dissections of polygons and planar $S$-dissections are obtained.
\end{abstract}

Keywords: dissections of a polygon; planar trees; unlabelled enumeration

\section{Introduction}

Enumeration of triangulations of a regular $(n+2)$-gon (or ways to dissect a convex $(n+2)$ gon into $n$ triangles by drawing $n-1$ diagonals, no two of which intersect in their interior) is one of the most well known problems in enumerative combinatorics. Apparently the first who have solved this problems in the case of a regular $(n+2)$-gon with a distinguished and oriented edge (equally, an $(n+2)$-gon with marked vertices) were Johann Andreas von Segner [1] and Leonhard Euler [2]. As a result of their decision they obtained the sequence

$$
1,1,2,5,14,42,132,429,1430,4862, \ldots,
$$

nowadays known as the sequence of Catalan numbers $C_{n}$ (sequence $A 000108$ in OEIS classification).

There is a big variety of combinatorial objects, enumeration of which can be described by $C_{n}$ (see for example [3]). In this article we will be especially interested in another equivalent combinatorial interpretation of these numbers, and namely, the number of trivalent plane trees with $n+2$ vertices of degree 1 , one of those being selected as a root (so-called trivalent planted plane trees). The correspondence between the dissections of an $(n+2)$-gon with a distinguished outer edge into triangles and such trees is shown in 


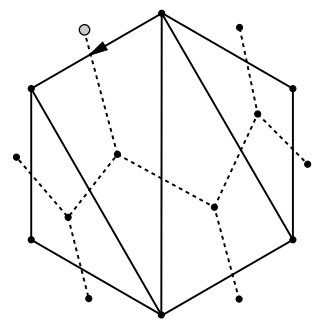

(a)

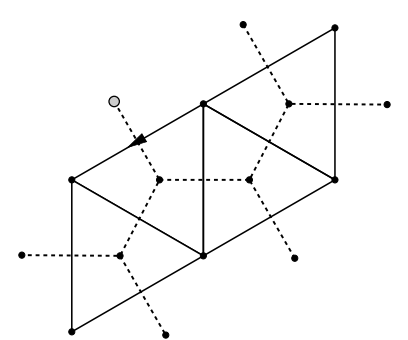

(b)

Figure 1: Dual structures

Figure 1(a). Instead of trivalent trees, their dual graphs - so-called plane 2-trees-are often enumerated [4],[5] (see Figure 1(b)).

Let us now consider the problem of counting the number of triangulations of a regular $(n+2)$-gon in the case when none of its edges is distinguished (so-called unrooted triangulations of polygons). The number of possible dissections will be reduced owing to internal symmetry of the polygon (Figure 2). Particularly, there is only one triangulation of a square $(n=2)$ and a pentagon $(n=3)$. Similarly, there are three or four different triangulations of a regular hexagon depending on the notion of the isomorphism of dissections we use, i.e. depending on the allowed isometries of the plane, which transform the hexagon into itself. There are four such dissections in case when two triangulations that differ only by a rotation are considered the same (so-called unrooted triangulations of polygons in the plane). And there are only three different triangulations of a $(n+2)$-gon in case when we allow not only rotations but reflections of polygon too (so called unrooted triangulations of polygons in space, also known as triangulations of the disc). Trivalent trees which correspond to these triangulations are shown in Figure 2 with dotted lines. In the terminology of Harary and Palmer [4] such trees are called plane and planar trees, respectively.
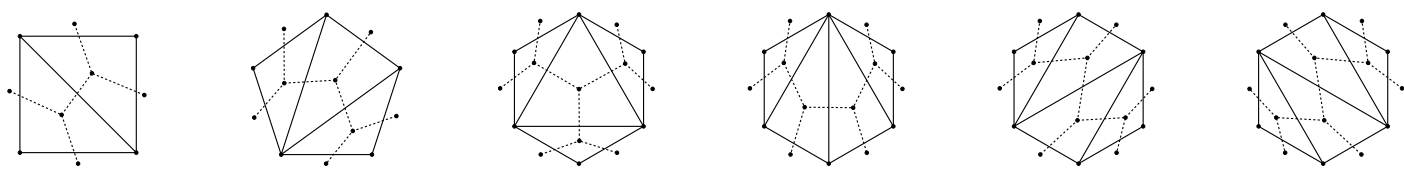

Figure 2: Triangulations for small values of $n$

The first solutions of the problems related to enumeration of unrooted triangulations (or, equivalently, enumeration of plane and planar trees) appeared only in the second half of the twentieth century. The numerical sequence which describes the number of unrooted triangulations of polygons in space was first obtained by Motzkin in the article [6]. In the articles [7], [8] and [9] some special methods of getting a simple analytical expression for the corresponding numbers were described. Harary and Palmer demonstrated in their book [4] how to construct the generating function that describes the number of planar 2 -trees and, consequently, the number of unrootes triangulations of $(n+2)$-gons in space. This approach was based on Redfield-Pólya theory $[10,11]$ and on Otter's dissimilarity 
theorem [12]. A year later, in 1974, Harary and Palmer [13] generalized the obtained result to the case of a regular $(n(r-2)+2)$-gon dissection into $n r$-gons or, in other words, they enumerated all the $r$-gonal planar 2-trees with $n$ vertices. However, the final expressions for the generating functions were rather cumbersome, what did not allow them to obtain explicit expressions for the numbers $V_{n}^{r}$ of those trees.

The theory of combinatorial species which appeared in the early 1980's stimulated progress in the solving the problems of counting planar trees [14],[15]. Reformulation of Otter's theorem [12] in terms of combinatorial species opened a route for elegant solutions for many problems concerning enumeration of tree-like structures. In paper [5] Labelle, Lamathe and Leroux presented the solution of the 2-tree counting problem in terms of the species theory and obtained molecular decomposition of those species. Also, the paper [16] should be mentioned, in which the solution of the problem of counting planar trees was used to enumerate polyene hydrocarbons with molecular formula $C_{n} H_{n+2}$.

Despite the impressive progress in solving the problems described above, the explicit formulas for the numbers of all unrooted dissections of polygons in space into $r$-gons have not been obtained yet. This is primarily due to the complexity of accounting for reflection symmetry in such problems. Rather simple formulas for generating functions that describe the number of dissections of a polygon into $r$-gons while accounting only for rotational symmetries were obtained in the [13]. Even more simple formulas for this case can be derived using the theory of combinational species. On the other hand, application of the dissymmetry theorem to the enumeration of polygon dissections while accounting for reflection symmetry makes the final results very complicated even in the simplest case of $r=3$ (triangulation of $(n+2)$-gons) [5].

Meanwhile, back in 1964, William G. Brown in his work [17] suggested another rather simple approach to accounting for reflection symmetry, which was based on the relationship between the number of unrooted dissections of polygons in the plane and in space. A much more complicated problem of counting the number of triangulations of a regular $(m+3)$-gon with $n$ internal points was considered in that paper. This problem for the case of triangulations with a distinguished oriented edge was first formulated and solved by William T. Tutte [18]. In the above-mentioned work [17] his student William G. Brown generalized the solution of this problem to the case of a regular $(m+3)$-gon triangulation without distinguished edge, i.e. accounting for all possible symmetries. He also showed in the article [19] that his approach can be effectively used to solve the counting problem of non-isomorphic dissections into quadrangles of a regular $(2 p+4)$-gon with $n$ internal points.

In the present paper we use the ideas expressed by Brown in the articles $[17,19]$ to prove the formulas (14) and (20) which link the number of the unrooted dissections of polygons in plane and in space.

\section{Enumeration of $r$-dissections}

Let us first consider a regular $(n(r-2)+2)$-gon. Adding non-intersecting chords to this polygon until each inner region becomes an $r$-gon, we finally get some dissection of the 
original polygon into $n r$-gons, which we will call an $r$-dissection. The generating function $f_{r}(x)$, which describes the number of dissections rooted at an oriented outer edge, satisfies the functional equation

$$
f_{r}(x)=1+x f_{r}^{r-1}(x) .
$$

This equation in rather well-known. In [13] it is stated as formula (2.2) in slightly different terms: we allow for a dissection to consist of zero inner regions, thus our $f_{r}(x)$ is equal to $U_{r}(x)+1$ in [13]. Using the Lagrange inversion theorem, one can obtain an explicit expression for the number $F_{n}^{(r)}$ of $r$-dissections from the equation (1):

$$
F_{n}^{(r)}=\frac{1}{n}\left(\begin{array}{c}
n(r-1) \\
n-1
\end{array}\right), \quad n>0 .
$$

In particular, in the case of $r=3$ we conclude that the number of triangulations of a regular $(n+2)$-gon rooted at an oriented outer edge is described by the Catalan numbers. In the general case we have the so-called Fuss-Catalan numbers [20].

Let us consider the same problem for unrooted polygons. We denote by $u_{r}(x)$ the generating function that describes the number $U_{n}^{(r)}$ of non-isomorphic dissections of a regular $(n(r-2)+2)$-gon into $r$-gons considering only rotational symmetry $(r$-dissections of unrooted polygons in the plane). This function can be expressed in terms of the generating function $f_{r}(x)$ :

$$
u_{r}(x)=x Z\left(C_{r} ; f_{r}(x)\right)+f_{r}(x)+\frac{f_{r}\left(x^{2}\right)-f_{r}^{2}(x)}{2} .
$$

Here, $Z\left(C_{r}\right)$ is the cyclic index series for the group $C_{r}$ of rotations of the regular $r$-gon,

$$
Z\left(C_{r} ; f_{r}(x)\right)=\frac{1}{r} \sum_{d \backslash r} \varphi(d) \cdot f_{r}^{r / d}\left(x^{d}\right),
$$

where $\varphi(d)$ is the Euler function. The proof of (3) can be found in [13], where it is stated as formula (4.3). Recall that $f_{r}(x)$ in the present article stands for $U_{r}(x)+1$ in [13], that is why the precise forms of the mentioned formulas slightly differ. We just note that the term $x Z\left(C_{r} ; f_{r}(x)\right)$ enumerates the dissections rooted at some $r$-gon, and this fact is proved using the famous Pólya theorem.

Using the formula (3) one can obtain the explicit expressions for the number of $r$ dissections of a regular $(n(r-2)+2)$-gon in the plane. As an example, for triangulations of a regular $(n+2)$-gon (case $r=3$ ) we have

$$
u_{3}(x)=\frac{2}{3} x f_{3}\left(x^{3}\right)+\frac{1}{2} f_{3}\left(x^{2}\right)+\frac{2}{3} f_{3}(x)-\frac{1}{6 x}\left(f_{3}(x)-1\right) .
$$

Hence it is easy to see that the number $U_{n}^{(3)}$ of triangulations of a regular $(n+2)$-gon considering rotational symmetries is given by

$$
U_{n}^{(3)}=\frac{1}{(n+2)} \cdot C_{n}+\frac{1}{2} \cdot C_{[n / 2]}+\frac{2}{3} \cdot C_{[(n-1) / 3]}
$$


(sequence $A 001683$ in OEIS). Here, $C_{[i]}$ is the Catalan number $C_{i}$ if $i$ is an integer, and zero otherwise.

Now let $v_{r}(x)$ be the generating function for the number of unrooted $r$-dissections of $(n(r-2)+2)$-gon in space. Our main objective is to find a simple expression for this function. Now we should take into account both rotational and reflective symmetries of the polygon. We will use the approach formulated by W. G. Brown in the articles [17] and [19].

First we define $s_{r}(x)$ to be the generating function for unrooted dissections that have at least one axis of symmetry. When we start to consider reflections, $u_{r}(x)$ still counts each dissection that has at least one axis of symmetry only once. But dissections which are not invariant under any reflection are counted by $u_{r}(x)$ twice: dissection and its reflected version are considered to be distinct. This is what we need to get rid of in $v_{r}(x)$. So, we add $s_{r}(x)$ to $u_{r}(x)$ and divide the result by two, thereby counting each dissection exactly once:

$$
v_{r}(x)=\frac{u_{r}(x)+s_{r}(x)}{2} .
$$

Brown introduced a tricky way to express $s_{r}(x)$ through generating functions for two special kinds of rooted dissections and stated it as Lemma 10.1 in [17] without proof. As this lemma is central for our enumeration scheme, we state it as Theorem 1 and give a proof of it.

First we introduce two generating functions, $H_{r}(x)$ and $K_{r}(x)$, and define them as functions which enumerate symmetric dissections rooted at a vertex and at an outer edge, respectively. By the word "symmetric" we mean that these dissections have an axis of symmetry that passes through the root, so that it is invariant under this symmetry.

Theorem 1. The following equality holds:

$$
s_{r}(x)=\frac{H_{r}(x)+K_{r}(x)}{2} .
$$

Proof. Let $M$ be the set of $r$-dissections that have at least one axis of symmetry. Each such dissection has a certain number $k$ of axes of symmetry and $2 k$ points, in which these axes intersect the boundary of a polygon. We call these points symmetry points. Clearly, symmetry points are located in vertices or in centers of outer edges. Dissections enumerated by $H_{r}(x)$ and $K_{r}(x)$ naturally correspond to dissections from $M$ rooted at their symmetry points. We claim that each dissection from $M$ can be rooted at its symmetry point in exactly two inequivalent ways.

In fact, consider an arbitrary dissection of such kind. Its symmetry group is the dihedral group $D_{k}$ for some $k$. Particularly, this dissection admits $k$ reflections and $k$ rotations (including trivial) and $2 k$ its symmetry points are located uniformly on its boundary. Due to rotational symmetry with period $2 \pi / k$, these points are divided into exactly two equivalence classes, each containing $k$ points. So, there are only two inequivalent ways of rooting, thus $H_{r}(x)+K_{r}(x)$ counts each dissection from $M$ twice. This completes the proof.

Now we substitute (5) into (4) and prove the following formula. 
Proposition 2. The generating function for $r$-dissections of a regular $(n(r-2)+2)$-gon is given by

$$
v_{r}(x)=\frac{u_{r}(x)}{2}+\frac{H_{r}(x)+K_{r}(x)}{4} .
$$

This proposition was originally proved by Brown in [17] for triangulations with internal points. It can be easily generalized to a big variety of different planar structures, since the proofs of (4) and (5) do not use any specific properties of $r$-dissections. To get explicit form of the generating function $v_{r}(x)$ from it, we have to derive formulas for $H_{r}(x)$ and $K_{r}(x)$. It turns out that there is a simple relation they satisfy.

Theorem 3. Half the sum of the generating functions $H_{r}(x)$ and $K_{r}(x)$ is expressed in terms of function $f_{r}(x)$ that describes the number of dissections rooted at an outer oriented edge, by the formula

$$
\frac{H_{r}(x)+K_{r}(x)}{2}=f_{r}\left(x^{2}\right)+x f_{r}^{\lfloor r / 2\rfloor}\left(x^{2}\right) .
$$

Proof. First, consider the case of $r$-dissections for odd $r, r=2 k+1, k=1,2, \ldots$ It is clear that the generating function $K_{r}(x)$ is expressed by the formula

$$
K_{r}(x)=1+x f_{r}^{k}\left(x^{2}\right) .
$$

Indeed, each symmetric dissection with a distinguished edge $a$ is either the trivial dissection consisting only of $a$, or has the form shown in Figure 3.

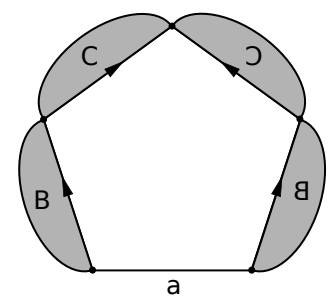

Figure 3: Symmetric dissection with a distinguished edge; $r=2 k+1$.

For calculating $H_{r}(x)$ in the case of $r=2 k+1$, we consider three types of dissections shown in Figure 4, differing in the number of $r$-gons crossed by the axis of symmetry. By considering these types, we conclude that the generating function $H_{r}(x)$ is given by

$$
H_{r}(x)=f_{r}\left(x^{2}\right)+x f_{r}^{k}\left(x^{2}\right)+x^{2} f_{r}^{2 k}\left(x^{2}\right) .
$$

Consequently, in the case of odd $r$, the half-sum of generating functions $H_{r}(x)$ and $K_{r}(x)$ is expressed as follows

$$
\frac{H_{r}(x)+K_{r}(x)}{2}=\frac{1+f_{r}\left(x^{2}\right)+2 x f_{r}^{k}\left(x^{2}\right)+x^{2} f_{r}^{2 k}\left(x^{2}\right)}{2} .
$$

But in virtue of equality (1)

$$
1+x^{2} f_{r}^{2 k}\left(x^{2}\right)=1+x^{2} f_{r}^{r-1}\left(x^{2}\right)=f_{r}\left(x^{2}\right) .
$$




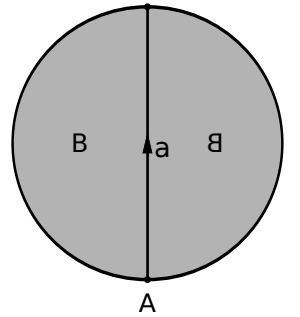

(a) First type

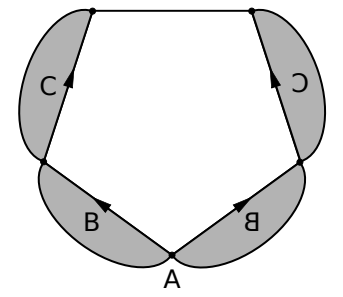

(b) Second type

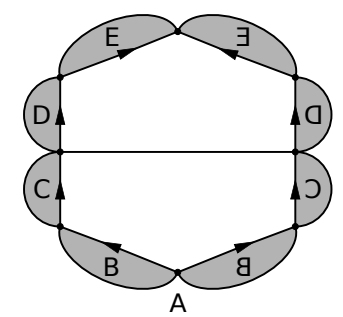

(c) Third type

Figure 4: Symmetric dissections with a distinguished vertex; $r=2 k+1$.

Therefore, finally, for half the sum of $H_{r}(x)$ and $K_{r}(x)$, in the case of $r=2 k+1$, we have

$$
\frac{H_{r}(x)+K_{r}(x)}{2}=f_{r}\left(x^{2}\right)+x f_{r}^{k}\left(x^{2}\right) .
$$

Now consider the case of $r=2 k, k=1,2, \ldots$ In this case, we consider the types of dissections shown in Figure 5 and conclude that the generating function $H_{r}(x)$ satisfies the equation

$$
H_{r}(x)=f_{r}\left(x^{2}\right)+x f_{r}^{k}\left(x^{2}\right)
$$

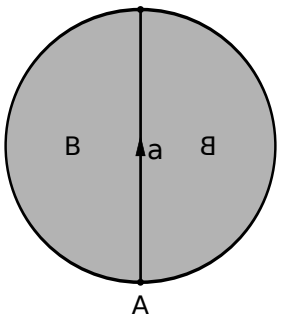

(a) First type

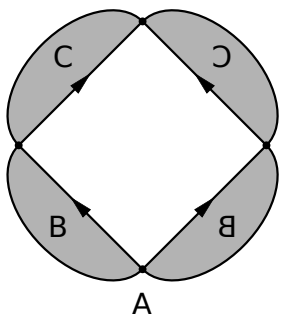

(b) Second type

Figure 5: Symmetric dissections with a distinguished vertex; $r=2 k$.

It remains to consider the most complicated case: that of a distinguished edge and even $r$. In this case, an arbitrary number of $r$-gons can be crossed by the axis of symmetry (Figure 6). Therefore, we obtain the expression for $K_{r}(x)$ in the form of an infinite sum

$$
K_{r}(x)=1+x f_{r}^{k-1}\left(x^{2}\right)+x^{2} f_{r}^{2 k-2}\left(x^{2}\right)+x^{3} f_{r}^{3 k-3}\left(x^{2}\right)+\ldots
$$

The right-hand side can be viewed as a geometric series with the first term $1+x f_{r}^{k-1}\left(x^{2}\right)$ and the common ratio $x^{2} f_{r}^{2 k-2}\left(x^{2}\right)$. Thus,

$$
K_{r}(x)=\frac{1+x f_{r}^{k-1}\left(x^{2}\right)}{1-x^{2} f_{r}^{2 k-2}\left(x^{2}\right)} .
$$

To further simplify this formula for $K_{r}(x)$, we derive another functional equation for $f_{r}(x)$ by subtracting $x f_{r}^{r-1}(x)$ from both sides of $(1)$ :

$$
f_{r}(x)\left(1-x f_{r}^{r-2}(x)\right)=1 \quad \Rightarrow \quad f_{r}(x)=\frac{1}{1-x f_{r}^{r-2}(x)}=\frac{1}{1-x f_{r}^{2 k-2}(x)} .
$$



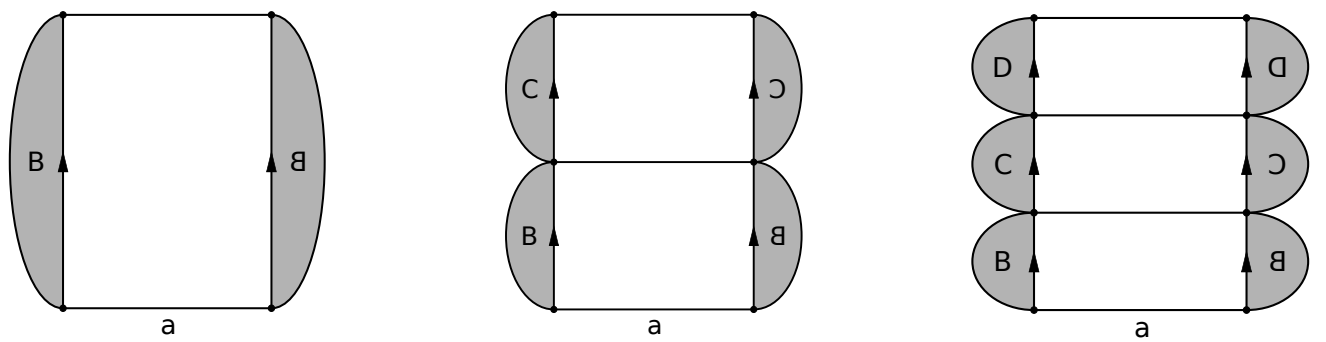

Figure 6: Symmetric dissections with a distinguished edge; $r=2 k$.

Taking this into account, we get rid of the division in the right-hand side of (12) and obtain the final form of the formula for $K_{r}(x)$ :

$$
K_{r}(x)=f_{r}\left(x^{2}\right)+x f_{r}^{k}\left(x^{2}\right) .
$$

Equality (13) can be proved by a purely combinatorial arguments. Consider the symmetric $2 k$-dissection with a distinguished edge in which the axis of symmetry intersects an even number of $r$-gons (Figure $7(\mathrm{a})$ ). We cut this dissection along the axis of symmetry $(\alpha, \alpha)$, as well as the axis $(\beta, \beta)$ perpendicular to it and passing through the central edge of the dissection. We rotate the lower-left and the upper-right quarter of the figure at $180^{\circ}$, thus swapping them, and then re-join the four pieces of dissection along the axes $(\alpha, \alpha)$ and $(\beta, \beta)$ (Figure $7(b))$. As a result we obtain a pair of dissections rooted at an outer edge and symmetric about the axis $(\beta, \beta)$, the number of which is described by the generating function $f_{r}\left(x^{2}\right)$.

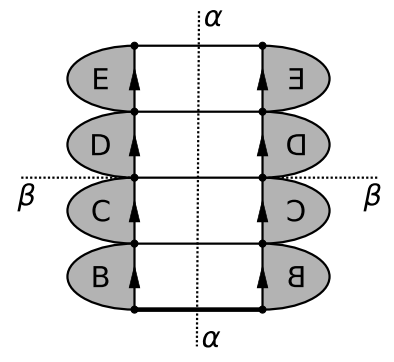

(a) Symmetric $2 k$-dissection

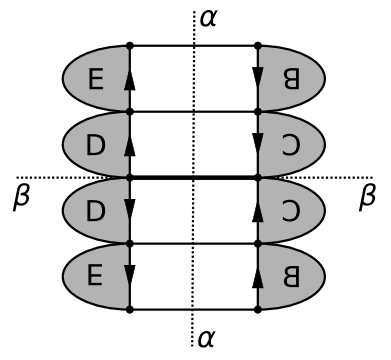

(b) A pair of equal $2 k$-dissections

Figure 7: For combinatorial proof of the equality (13).

If the axis of symmetry crosses an odd number of $r$-gons, we account one of them (say, the one with a distinguished edge) separately. Together with $k-1$ pairs of dissections attached to its sides, it is enumerated by $x f_{r}^{k-1}\left(x^{2}\right)$. The remaining part of a dissection has an even number of $r$-gons intersected by the axis of symmetry, and is enumerated by $f_{r}\left(x^{2}\right)$, as already proved. Equality (13) follows immediately.

In view of the expressions (10) and (13) for the case of $r=2 k$ we obtain

$$
\frac{H_{r}(x)+K_{r}(x)}{2}=f_{r}\left(x^{2}\right)+x f_{r}^{k}\left(x^{2}\right) .
$$


Substituting expression (7) in Brown's formula (6), and recalling the explicit expression (3) for the function $u_{r}(x)$, we obtain the following final result.

Consequence 4 . The generating function $v_{r}(x)$ for the number of $r$-dissections regarding both the reflections and rotations is calculated by the formula:

$$
\begin{aligned}
v_{r}(x) & =\frac{u_{r}(x)}{2}+\frac{f_{r}\left(x^{2}\right)+x f_{r}^{\lfloor r / 2\rfloor}\left(x^{2}\right)}{2}= \\
& =\frac{1}{2} f_{r}(x)-\frac{1}{4} f_{r}^{2}(x)+\frac{3}{4} f_{r}\left(x^{2}\right)+\frac{x}{2} f_{r}^{\lfloor r / 2\rfloor}\left(x^{2}\right)+\frac{x}{2 r} \sum_{d \backslash r} \varphi(d) f_{r}^{r / d}\left(x^{d}\right) .
\end{aligned}
$$

The formula (14) makes it quite easy to express the number $V_{n}^{(r)}$ of all the dissections of a regular $(n(r-2)+2)$-gon into $n r$-gons in terms of the coefficients

$$
\begin{gathered}
B(n, r, k)=\frac{k}{n}\left(\begin{array}{c}
n(r-1)+(k-1) \\
n-1
\end{array}\right), \quad n \in \mathbb{Z}_{+} ; \\
B(0, r, k)=1 ; \quad B(n, r, k)=0, \quad n \notin \mathbb{Z}_{+} \cup\{0\},
\end{gathered}
$$

which generalize Fuss-Catalan numbers $F_{n}^{(r)}=B(n, r, 1)$, for any fixed value of the parameter $r$. Indeed, application of the Lagrange-Bürmann formula to (1) allows us to conclude that the coefficient of $x^{n}$ in $f_{r}^{k}(x)$ is equal to $B(n, r, k)$. Thus, for prime $r$ we have:

$$
V_{n}^{(r)}=\frac{B(n, r, 1)}{2(n(r-2)+2)}+\frac{(r-1) B\left(\frac{n-1}{r}, r, 1\right)}{2 r}+\frac{B\left(\frac{n-1}{2}, r,\lfloor r / 2\rfloor\right)}{2}+\frac{3 B(n / 2, r, 1)}{4} .
$$

\section{Enumeration of $S$-dissections}

Now we are going to demonstrate the effectiveness of this approach by enumerating the dissections in which the number of edges of each inner face belongs to a predefined set.

Definition 5. Let $S$ be any subset of $\mathbb{Z}_{+} \backslash\{1,2\}$, where $\mathbb{Z}_{+}$denotes the set of all positive integers. We define an $S$-dissection as a dissection, any inner face of which is a polygon with the number of edges belonging to the set $S$.

An example of a $\{3,4,8\}$-dissection is given in Figure 8. Note that this specific $\{3,4,8\}$ dissection has no octagonal faces.

To enumerate $S$-dissections regarding all the symmetries, we first consider rooted plane $S$-dissections. The problem of enumerating the trees dual to those dissections is described in detail in [3]. In contrast to $r$-dissections, we enumerate $S$-dissections not by the number of inner regions, but by the number of outer edges. Let $g_{S}(x)$ be the generating function for the number $G_{n}$ of plane $S$-dissections rooted at an oriented outer edge and having $n+1$ outer edges in total. It is determined by the functional equation

$$
g_{S}(x)=x+\sum_{i \in S} g_{S}^{i-1}(x) .
$$




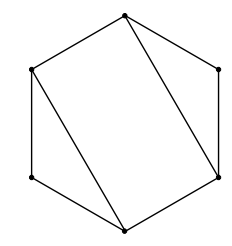

Figure 8: $\{3,4,8\}$-dissection.

The following formula for the number of plane $S$-dissections can be derived either in the same manner as (3) or by applying the dissymmetry theorem [15, Ch. 4].

Proposition 6. Let $u_{S}(x)$ be the ordinary generating function which describes the number of all plane $S$-dissections with $n$ outer edges. Then,

$$
u_{S}(x)=\frac{g_{S}\left(x^{2}\right)-g_{S}^{2}(x)}{2}+x \cdot g_{S}(x)+\sum_{i \in S} Z\left(C_{i} ; g_{S}(x)\right) .
$$

Proof. Observing the bijection between unrooted trees and unrooted dissections shown in Figure 2, one can establish the correspondence between vertex-rooted trees and dissections that are rooted either at an outer edge or at a polygon, depending on whether leaf or non-leaf was distinguished in a tree. For these kinds of trees we introduce the generating function $q(x)$. Edge-rooted trees always correspond to edge-rooted dissections. They are enumerated by $\left(g_{S}\left(x^{2}\right)+g_{S}^{2}(x)\right) / 2$. Pairs of dissections rooted at an oriented outer edge are given by $g_{S}^{2}(x)$. Combining these observations, one can rewrite the dissymmetry theorem for dissections as follows:

$$
q(x)+\frac{g_{S}\left(x^{2}\right)+g_{S}^{2}(x)}{2}=u_{S}(x)+g_{S}^{2}(x) .
$$

Applying Pólya theorem to find $q(x)$, one can get that $q(x)=\sum_{i \in S} Z\left(C_{i} ; g_{S}(x)\right)+x \cdot g_{S}(x)$. Equality (16) follows from (17) after some simplification.

Now we are going to use Brown's approach to enumerate $S$-dissections regarding all of the symmetries. Let us denote by $v_{S}(x)$ the generating function for planar dissections. Our goal is to express $v_{S}(x)$ in terms of $u_{S}(x)$ and $g_{S}(x)$. First, one can see, that Brown's formula also holds for the generating function $v_{S}(x)$ for planar $S$-dissections with $n$ outer edges:

$$
v_{S}(x)=\frac{u_{S}(x)}{2}+\frac{H_{S}(x)+K_{S}(x)}{4} .
$$

Here, the generating functions $H_{S}(x)$ and $K_{S}(x)$ correspond to $S$-dissections of a regular $n$-gon with a distinguished vertex or outer edge, respectively, lying on one of the axes of symmetry. To enumerate planar $S$-dissections, we should find the expressions for those generating functions.

First we introduce the following auxiliary generating functions: 


$$
R_{S}(x)=\sum_{i \in S_{\text {odd }}} g_{S}^{(i-1) / 2}\left(x^{2}\right) \quad \text { and } \quad Q_{S}(x)=\sum_{i \in S_{\text {even }}} g_{S}^{i / 2-1}\left(x^{2}\right) .
$$

Here, $S_{\text {odd }}$ and $S_{\text {even }}$ are the sets consisting of, respectively, odd and even elements of set $S$. Let us describe the types of dissections the functions $R_{S}(x)$ and $Q_{S}(x)$ enumerate.

Dissections that have $n+1$ outer edges, a distinguished outer edge $a$ lying on the axis of symmetry, and a single $i$-gon with odd number of edges crossed by this axis (Figure 9(a)), are enumerated by $R_{S}(x)$. Summation in the expression for $R_{S}(x)$ stands for looking over all the possible $i$-gons on the axis of symmetry. For a fixed $i$, we have to choose $(i-1) / 2$ pairs of $S$-dissections, and glue them to the edges of a central $i$-gon. To preserve the symmetry, each such pair should consist of two dissections, which are mirror images of each other. Clearly, the generating function for each pair is $g_{S}\left(x^{2}\right)$, and the given expression for $R_{S}(x)$ immediately follows.

$Q(x)$ enumerates similar structures, with the only difference, that the central $i$-gon has even number of edges (Figure 9(b)), and the total number of outer edges is $n+2$.

The representative of the other type of dissections also enumerated by $R_{S}(x)$ is shown in Figure 9(c). Dissections of this type have $n+1$ outer edges, a distinguished vertex $A$ lying on the axis of symmetry, and a single $i$-gon $(i=2 k+1)$ crossed by this axis.

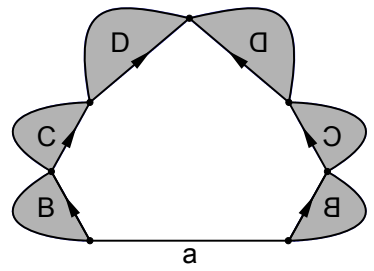

(a) $R_{S}(x)$

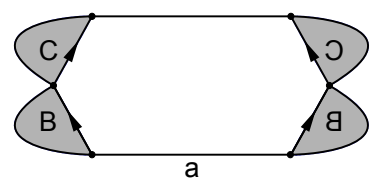

(b) $Q_{S}(x)$

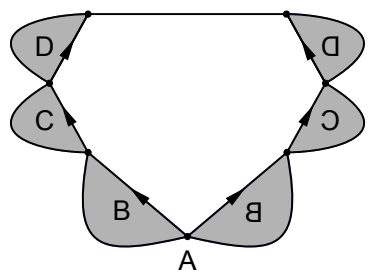

(c) $R_{S}(x)$

Figure 9: Types of dissections enumerated by $Q_{S}(x)$ and $R_{S}(x)$.

Now we claim that

$$
K_{S}(x)=x \cdot \frac{1}{1-Q_{S}(x)} \cdot\left(x+R_{S}(x)\right) .
$$

Indeed, each $S$-dissection, rooted at an outer edge that lies on the axis of symmetry (Figure 10), consists of three parts. The first part is an outer edge that is a root. It is enumerated just by $x$. The second part is a (possibly empty) sequence of structures, each of which is enumerated by $Q_{S}(x)$. The generating function for the whole sequence is $1+Q_{S}(x)+Q_{S}^{2}(x)+\cdots=1 /\left(1-Q_{S}(x)\right)$. The third part is either an outer edge (Figure 10(a)) or an odd-sided polygon with pairs of dissections glued to it (Figure 10(b)). As we already observed, the latter case corresponds to generating function $R_{S}(x)$.

We also claim that

$$
H_{S}(x)=g_{S}\left(x^{2}\right)+\sum_{i \in S_{\text {even }}} g_{S}^{i / 2}\left(x^{2}\right)+R_{S}(x) \frac{K_{S}(x)}{x} .
$$




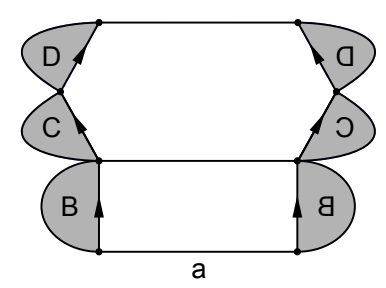

(a) First type

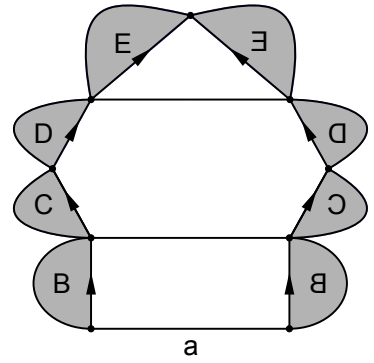

(b) Second type

Figure 10: Types of $S$-dissections with a distinguished edge.

To prove this statement, we observe that dissections with a distinguished vertex that lies on the axis of symmetry, naturally fall into three groups. Each group corresponds to one summand in the equality. The first group consists of dissections that have an edge coinciding with the axis of symmetry (Figure 11(a)). These structures are enumerated by $g_{S}\left(x^{2}\right)$. Dissections from the second group have only one $2 k$-gon on the axis of symmetry (Figure 11(b)). This group is enumerated by $\sum_{i \in S_{\text {even }}} g_{S}^{i / 2}\left(x^{2}\right)$, as the number of sides $i=2 k$ of the central polygon can be arbitrary even number from set $S$. Finally, the third group consists of the dissections in which the distinguished vertex is a vertex of the polygon that is crossed by the axis of symmetry, and has an odd number of sides (Figure 11(c)). This polygon, together with pairs of dissections, attached to it, is described by $R_{S}(x)$, as observed previously. The remaining part of the dissection can be viewed as a dissection rooted at outer edge, which is described by $K_{S}(x)$. Division by $x$ accounts that the root of this dissection is not outer, but inner edge in the resulting structure.

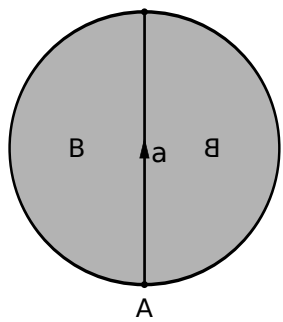

(a) First type

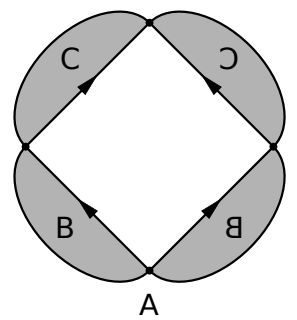

(b) Second type

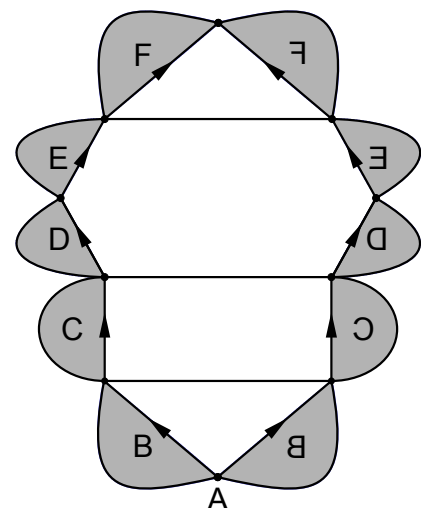

(c) Third type

Figure 11: Symmetric $S$-dissections with a distinguished vertex.

Now we can substitute the obtained expressions for $H_{S}(x)$ and $K_{S}(x)$ into equation (18) and obtain the following result.

Theorem 7. The generating function $v_{S}(x)$ for planar $S$-dissections enumerated by the 
number of outer edges satisfies the following equality:

$$
v_{S}(x)=\frac{u_{S}(x)}{2}+\frac{g_{S}\left(x^{2}\right)+\sum_{i \in S_{\text {even }}} g_{S}^{i / 2}\left(x^{2}\right)+\left(x+R_{S}(x)\right)^{2} /\left(1-Q_{S}(x)\right)}{4} .
$$

Now we are going to consider some special values of $S$. The formula (20) has the simplest form in the case $S_{\text {even }}=\varnothing$. For such $S$

$$
v_{S}(x)=\frac{u_{S}(x)}{2}+\frac{g_{S}\left(x^{2}\right)+\left(x+R_{S}(x)\right)^{2}}{4} .
$$

Consider, for example, $S=\{3,5\}$. For $\{3,5\}$-dissections, $g_{S}(x)$ satisfies the following functional equation:

$$
g_{S}(x)=x+g_{S}^{2}(x)+g_{S}^{4}(x) .
$$

The function $R_{S}(x)$ is given by

$$
R_{S}(x)=g_{S}\left(x^{2}\right)+g_{S}^{2}\left(x^{2}\right)=g_{S}\left(x^{2}\right)\left(1+g_{S}\left(x^{2}\right)\right),
$$

and the generation function for planar $\{3,5\}$-dissections is equal to

$$
\begin{gathered}
v_{S}(x)=\frac{u_{S}(x)}{2}+g_{S}\left(x^{2}\right) \frac{1+x+x \cdot g_{S}\left(x^{2}\right)+g_{S}^{2}\left(x^{2}\right)}{2}= \\
=\frac{g_{S}\left(x^{3}\right)}{3}+\frac{2 g_{S}\left(x^{5}\right)}{5}-\frac{3 g_{S}^{2}(x)}{20}+\frac{2 x g_{S}(x)}{5}+\frac{g_{S}^{3}(x)}{15}+g_{S}\left(x^{2}\right) \frac{3+2 x+2 x g_{S}\left(x^{2}\right)+2 g_{S}^{2}\left(x^{2}\right)}{4} .
\end{gathered}
$$

\begin{tabular}{c|ccccc}
\hline$n \backslash S$ & $\{3,5\}$ & $\{3,4\}$ & $\{4,6,8, \ldots\}$ & $\{3,5,7, \ldots\}$ & $\{3,4,5, \ldots\}$ \\
\hline 3 & 1 & 1 & 0 & 1 & 1 \\
4 & 1 & 2 & 1 & 1 & 2 \\
5 & 2 & 2 & 0 & 2 & 3 \\
6 & 4 & 7 & 2 & 4 & 9 \\
7 & 7 & 14 & 0 & 8 & 20 \\
8 & 22 & 53 & 4 & 23 & 75 \\
9 & 60 & 171 & 0 & 65 & 262 \\
10 & 208 & 691 & 13 & 223 & 1117 \\
11 & 695 & 2738 & 0 & 757 & 4783 \\
12 & 2566 & 11720 & 48 & 2824 & 21971 \\
13 & 9451 & 50486 & 0 & 10559 & 102249 \\
14 & 36158 & 224012 & 238 & 40994 & 489077 \\
15 & 139574 & 1005468 & 0 & 160734 & 2370142 \\
\hline
\end{tabular}

Table 1: The numbers of planar $S$-dissections of $n$-gon. 
Formula (20) also allows us to count the number of $S$-dissections in the case when $S$ is infinite. So, one can consider the dissections of a polygon into odd-sided polygons:

$$
S=\{3,5,7,9,11, \ldots\} \text {. }
$$

In this case

$$
Q_{S}(x)=0, \quad R_{S}(x)=g_{S}\left(x^{2}\right) /\left(1-g_{S}\left(x^{2}\right)\right),
$$

and the generating function for rooted dissections is described by the functional equation

$$
g_{S}(x)=x+g_{S}^{2}(x)+g_{S}^{4}(x)+\ldots=x+g_{S}^{2}(x) /\left(1-g_{S}^{2}(x)\right) .
$$

Using this equation, it is possible to simplify the expression for $R_{S}(x)$ :

$$
R_{S}(x)=2 g_{S}\left(x^{2}\right)+g_{S}^{2}\left(x^{2}\right)-x^{2}-x^{2} g_{S}\left(x^{2}\right) .
$$

As a consequence, we have the following equation for the generating function for the dissections of a polygon into odd-sided polygons:

$$
v_{S}(x)=\frac{u_{S}(x)}{2}+\frac{g_{S}\left(x^{2}\right)+\left(2 g_{S}\left(x^{2}\right)+g_{S}^{2}\left(x^{2}\right)-x^{2}-x^{2} g_{S}\left(x^{2}\right)+x\right)^{2}}{4} .
$$

Finally, we consider the most general case

$$
S=\{3,4,5, \ldots\}=\mathbb{Z}_{+} \backslash\{1,2\} .
$$

For this kind of $S$, the rooted dissections are enumerated by the function

$$
g_{S}(x)=x+g_{S}^{2}(x)+g_{S}^{3}(x)+\ldots=x+g_{S}^{2}(x) /\left(1-g_{S}(x)\right) .
$$

The number $G_{n}$ of such dissections is nothing else than small Schroeder numbers (sequence $A 001003$ in OEIS). For $v_{S}(x)$ we have the following relation:

$$
v_{S}(x)=\frac{u_{S}(x)}{2}+\frac{R_{S}(x)+\left(R_{S}(x)+x\right)^{2} /\left(1-R_{S}(x)\right)}{4}, \quad R_{S}(x)=2 g_{S}\left(x^{2}\right)-x^{2} .
$$

The corresponding numerical sequence describes the number of arbitrary planar dissections of $(n+2)$-gon (sequence $A 001004$ in OEIS).

The first terms for three considered special cases of $S$ and for two others can be found in Table 1. They were computed using the obtained expressions for $v_{S}(x)$.

\section{Asymptotics}

To obtain the asymptotic estimates for the number of $S$-trees we follow [21, Ch. 7]. One can see that (15) can be rewritten as

$$
g_{S}(x)=\frac{x}{1-\sum_{i \in S} g_{S}^{i-2}(x)}=x \cdot \phi\left(g_{S}(x)\right),
$$


where $\phi(x)=1 /\left(1-\sum_{i \in S} x^{i-2}\right)$. Now, let $S$ be equal to $\{3,5\}$. Then it can be easily verified, that $g_{S}(x)$ belongs to the smooth inverse-function schema in the aperiodic case (in terms of $[21$, Ch. 7]), and therefore,

$$
\left[x^{n}\right] g_{S}(x)=\sqrt{\frac{\phi(\tau)}{2 \phi^{\prime \prime}(\tau)}} \frac{\rho^{-n}}{\sqrt{\pi n^{3}}}(1+O(1 / n)) .
$$

Here, $\tau$ is the unique positive solution of $\phi(\tau)-\tau \cdot \phi^{\prime}(\tau)=0$ that lies within the open disc of convergence of $\phi$ at 0 , and $\rho=\tau / \phi(\tau)$. One can compute the values $\tau=0.3854584 \ldots$ and $\rho=0.2148047 \ldots$ As a consequence,

$$
\left[x^{n}\right] g_{S}(x) \sim A \cdot n^{-3 / 2} \cdot C^{n},
$$

where $A=0.0950642 \ldots, C=4.6553906 \ldots$ Recall that this is the asymptotic estimate for the number of $S$-dissections rooted at an oriented outer edge, which have $n+1$ outer edges in total. But we are interested in $\left[x^{n}\right] v_{S}(x)$, the number of planar $S$-dissections with $n$ outer edges. It is easy to see that, as $n$ tends to infinity, the ratio of the number of asymmetric planar dissections to the number of all planar dissections tends to 1 (almost all dissections are asymmetric). Each asymmetric planar dissection corresponds to $2 n$ rooted dissections, thus

$$
\left[x^{n}\right] v_{S}(x) \sim \frac{\left[x^{n-1}\right] g_{S}(x)}{2 n} .
$$

We finally obtain the following asymptotic estimate for the case $S=\{3,5\}$ :

$$
\left[x^{n}\right] v_{S}(x) \sim B \cdot n^{-5 / 2} \cdot C^{n}, \text { where } B=\frac{A}{2 C}=0.0102101 \ldots, C=4.6553906 \ldots .
$$

The same computations can be performed for three out of four remaining considered cases: $S=\{3,4\}, S=\{3,5,7, \ldots\}$ and $S=\{3,4,5, \ldots\}$.

The case $S=\{4,6,8, \ldots\}$ needs a little bit more attention, because it corresponds to the function $\phi(x)=\left(x^{2}-1\right) /\left(2 x^{2}-1\right)$, which is periodic (in the sense of [21, Ch. 4]). We have

$$
g_{S}(x)=x+\frac{g_{S}^{3}(x)}{1-g_{S}^{2}(x)} .
$$

Let $\tilde{g}_{S}(x)=g_{S}(\sqrt{x}) / \sqrt{x}-1$ (thus, $\left[x^{k}\right] \tilde{g}_{S}(x)=\left[x^{2 k+1}\right] g_{S}(x)$ for $\left.k>0\right)$. The generating function $\tilde{g}_{S}(x)$ satisfies

$$
\tilde{g}_{S}(x)=x \cdot\left(2 \cdot\left(\tilde{g}_{S}(x)+1\right)^{3}-\left(\tilde{g}_{S}(x)+1\right)^{2}\right),
$$

again belongs to the smooth inverse-function schema, but now the corresponding function $\phi(x)=2 \cdot(x+1)^{3}-(x+1)^{2}$ is aperiodic. Applying general method, we find the asymptotics for the coefficients of $\tilde{g}_{S}(x)$ :

$$
\left[x^{n}\right] \tilde{g}_{S}(x) \sim A \cdot n^{-3 / 2} \cdot D^{n}, \text { where } A=0.1931607 \ldots, D=8.8182997 \ldots
$$


Turning back to the function $g_{S}(x)$, one can obtain:

$$
\left[x^{n}\right] g_{S}(x) \sim \frac{\left[x^{n / 2-1}\right] \tilde{g}_{S}(x)}{2 n} \sim B \cdot n^{-5 / 2} \cdot C^{n}
$$

where $B=A \sqrt{2} / D=0.0309776 \ldots, C=\sqrt{D}=2.9695622 \ldots$, and $n$ is even.

So, we have asymptotics in the form $B \cdot n^{-5 / 2} \cdot C^{n}$ for all five considered types of $S$-dissections. The corresponding values of $B$ and $C$ are summarized in the Table 2.

\begin{tabular}{c|ccccc}
\hline$S$ & $\{3,5\}$ & $\{3,4\}$ & $\{4,6,8, \ldots\}$ & $\{3,5,7, \ldots\}$ & $\{3,4,5, \ldots\}$ \\
\hline$B$ & $0.0102101 \ldots$ & $0.0079480 \ldots$ & $0.0309776 \ldots$ & $0.0090465 \ldots$ & $0.0059602 \ldots$ \\
$C$ & $4.6553906 \ldots$ & $5.4000000 \ldots$ & $2.9695622 \ldots$ & $4.7305769 \ldots$ & $5.8284271 \ldots$ \\
\hline
\end{tabular}

Table 2: The coefficients of the asymptotic estimates for the number of $S$-dissections.

\section{Conclusion}

The problem of enumerating unrooted planar $r$-dissections is rather well-known. Harary solved this problem [13] for arbitrary $r$ and noted that the number of planar $r$-dissections is expressed through the number of plane $r$-dissections in two different ways, depending on the parity or $r$. In this article it is shown that, by using Brown's approach, one can obtain a surprisingly simple formula (7) that unifies these two cases. The corresponding approach has also been used for enumerating $S$-dissections for arbitrary $S$ and led to the formula (20) that can be used for computing the number of those dissections. Asymptotic estimates for the growth of the corresponding sequences are given for some special values of $S$.

\section{References}

[1] A. Segner. Enumeratio modorum quibus figurae planae rectilineae per diagonales dividuntur in triangula. Novis Commentar. Petropolit., 7:203-209, 1758.

[2] L. Euler. Summarium. Novis Commentar. Petropolit., 7:13-15, 1758.

[3] R. Stanley. Enumerative combinatorics. Vol. 2. Cambridge University Press, 2001.

[4] F. Harary and E. M. Palmer. Graphical enumeration. Academic Press, New York, 1973.

[5] G. Labelle, C. Lamathe, and P. Leroux. A classification of plane and planar 2-trees. Theoretical Computer Science, 307:337-363, 2003.

[6] T. S. Motzkin. Relations between hypersurface cross ratios and a combinatorial formula for partitions of a polygon, for permanent preponderance and for non-associative products. Bull. Amer. Math. Soc., 54:352-360, 1948. 
[7] R. K. Guy. Dissecting a Polygon Into Triangles. Research Paper No. 9. The University of Calgary, Department of Mathematics, 1967.

[8] J. W. Moon and L. Moser. Triangular dissections of n-gons. Canad. Math. Bull., 6(175-178), 1963.

[9] P. K. Stockmeyer. The charm bracelet problem and its applications. In R. A. Bari and F. Harary, editors, Graphs and Combinatorics, volume 406, pages 339-349. SpringerVerlag, 1973.

[10] H. J. Redfield. The theory of group-reduced distributions. American Journal of Mathematics, 49(3):433-455, 1927.

[11] G. Pólya and R. C. Read. Combinatorial Enumeration of Groups, Graphs, and Chemical Compounds. New York: Springer-Verlag, 1987.

[12] R. Otter. The number of trees. Ann. Math., 49:583-599, 1948.

[13] F. Harary, E. M. Palmer, and R. C. Read. On the cell-growth problem for arbitrary polygons. Discrete Mathematics, 11:371-389, 1975.

[14] André Joyal. Une théorie combinatoire des séries formelles. Advances in Mathematics, 42:1-82, 1981.

[15] F. Bergeron, G. Labelle, and P. Leroux. Combinatorial Species and Tree-Like Structures. Cambridge University Press, 1998.

[16] S. J. Cyvin, J. Brunvoll, E. Brendsdal, B. N. Cyvin, and E. K. Lloyd. Enumeration of polyene hydrocarbons: a complete mathematical solution. J. Chem. Inform. Comput. Sci., 35(4):743-751, 1995.

[17] W. G. Brown. Enumeration of triangulations of the disk. Proc. London Math. Soc., 14(3):746-768, 1964.

[18] W. T. Tutte. A census of planar triangulations. Canadian J. Math., 14:21-38, 1962.

[19] W. G. Brown. Enumeration of quadrangular dissections of the disk. Canad. J. Math., 17:302-317, 1965.

[20] R. L. Graham, D. E. Knuth, and O. Patashnik. Concrete Mathematics. AddisonWesley, Reading, MA, 1990.

[21] P. Flajolet and R. Sedgewick. Analytic Combinatorics. Cambridge University Press, Cambridge, UK, 2009. 